\title{
Trasplante hepático en hemangioendotelioma epitelioide: reporte de dos casos y revisión de la literatura
}

\section{Two Case Reports and a Literature Review of Liver Transplantation for Epithelioid Hemangioendothelioma}

\author{
Juan Ignacio Marín, MD, ${ }^{1}$ Óscar Santos, MD, ${ }^{1}$ Octavio Muñoz, MD, ${ }^{1}$ Germán Osorio, MD, ${ }^{2}$ Carlos Guzmán, MD, ${ }^{1}$ Sergio Hoyos, MD, \\ Álvaro Mena, MD, ${ }^{1}$ Juan Carlos Restrepo, MD. ${ }^{1}$
}

\footnotetext{
Unidad de Hepatología y Trasplante hepático, Hospital Pablo Tobón Uribe/Universidad de Antioquia. Medellín, Colombia.

2 Departamento de Patología, Universidad de Antioquia. Medellín, Colombia.
}

Fecha recibido: $28-05-14$ Fecha aceptado: 06-04-15

\begin{abstract}
Resumen
El hemangioendotelioma epitelioide hepático es un tumor raro, de naturaleza agresiva y comportamiento impredecible, con una incidencia de menos de 1 caso por millón de personas al año. Se presenta especialmente en mujeres jóvenes y con síntomas no específicos como dolor abdominal y pérdida de peso. La presentación radiológica es con múltiples nódulos o masas periféricas y bilaterales, con retracción de la cápsula, en algunos casos con calcificaciones y compromiso vascular. Debe realizarse biopsia hepática para confirmar el diagnóstico y para descartar diagnósticos alternativos como angiosarcoma, hepatocarcinoma, colangiocarcinoma y metástasis. El manejo depende de la extensión al momento de presentación, aunque solo el $10 \%$ de los casos es susceptible de resección. En las últimas décadas, el trasplante hepático se ha convertido en una opción de manejo, con series de casos aisladas reportadas por centros de trasplante en el mundo. En este artículo se presenta la experiencia de 2 pacientes con diagnóstico de hemangioendotelioma epitelioide hepático sin posibilidad de resección, confirmado histológicamente, sin compromiso extrahepático y que fueron llevados a trasplante de hígado exitoso y sin recurrencia de la enfermedad a 4 y 6 años después del trasplante.
\end{abstract}

\section{Palabras clave}

Trasplante hepático, hemangioendotelioma epitelioide hepático, tumor hepático.

\begin{abstract}
Hepatic epithelioid hemangioendothelioma is a rare, aggressive and unpredictable tumor. Its incidence is less than one case per million people per year. Most commonly, it occurs in young women who exhibit nonspecific symptoms such as abdominal pain and weight loss. Radiologically it presents as multiple bilateral and peripheral nodules or masses with retraction of the capsule of the liver. In some cases there is calcification and vascular compromise. A liver biopsy should be performed to confirm the diagnosis and to rule out alternative diagnoses such as angiosarcoma, hepatocellular carcinoma, cholangiocarcinoma and metastasis. Management depends on the extension at presentation, but only $10 \%$ of cases are amenable to resection. Although in recent decades liver transplantation has become a management option, only studies of isolated cases reported by various transplant centers around the world have been published so far. In this article, we report our experience with two patients who were diagnosed with hepatic epithelioid hemangioendothelioma in which there were no possibilities of resection. Diagnoses were histologically confirmed, and since the patients had no extra-hepatic compromises, liver transplantations were performed. Both were successful and without recurrence at 4 and 6 years after transplantation.
\end{abstract}

\section{Keywords}

Liver transplantation, hepatic epithelioid hemangioendothelioma, liver tumor. 


\section{INTRODUCCIÓN}

El hemangioendotelioma epitelioide (HEE) hepático es un tumor raro, de origen vascular, que compromete los tejidos blandos y diferentes órganos específicos $(1,2)$, con una incidencia de menos de 1 caso por millón de personas al año. La patogénesis es desconocida, aunque hay datos de asociación con el uso de anticonceptivos orales (3), exposición a cloruro de vinilo (4) e infección previa por el virus de la hepatitis B (VHB) y el virus de la hepatitis C (VHC) (5).

El HEE fue reportado por primera vez por Ishak, en 1984, en una serie de 32 pacientes (2-6). Desde entonces, ha sido descrito en órganos como el bazo, el hueso, las meninges, la mama, el corazón, el estómago y los nódulos linfáticos (6). Afecta comúnmente a mujeres y se caracteriza por una morfología epitelioide o histiocitoide y un patrón de crecimiento con evidencia de histogénesis endotelial (5). El HEE tiene un curso clínico y potencial maligno impredecible, con comportamiento entre hemangioma y angiosarcoma, siendo el diagnóstico diferencial con este último muy difícil, por lo que se requieren hallazgos específicos en la inmunohistoquímica para confirmar el diagnóstico (7). Debido a su naturaleza clínica y evolución variable, no existe un consenso en el tratamiento de estos pacientes $(8,9)$. El tratamiento primario de este tipo de lesiones es la resección quirúrgica, aunque generalmente el diagnóstico es tardío con enfermedad avanzada, razón por la que se consideran otras opciones como quimioterapia, radioterapia, trasplante hepático (en algunos casos) y hasta en un $25 \%$ de los pacientes no se lleva a cabo tratamiento alguno (2-9). Nuestro objetivo es reportar la experiencia con el trasplante de hígado en 2 pacientes con HEE hepático y hacer una revisión de la literatura.

\section{CASOS CLÍNICOS}

\section{Caso n. ${ }^{0} 1$}

Paciente de 36 años de edad, sexo femenino, sin antecedentes personales de importancia, sin consumo de medicamentos ni exposición a tóxicos. Remitida al programa de trasplante hepático en el 2006 por cuadro clínico de 6 meses de dolor abdominal y aumento del perímetro, con pérdida de peso. Las pruebas de laboratorio muestran: AST, 54 $\mathrm{U} / \mathrm{L}$; ALT, $36 \mathrm{U} / \mathrm{L}$; bilirrubina total, $1,2 \mathrm{mg} / \mathrm{dL}$; fosfatasa alcalina, $382 \mathrm{U} / \mathrm{L}$; gamma-glutamil transpeptidasa, 155 $\mathrm{U} / \mathrm{L}$; albúmina, 3,0 g/dL; alfa-fetoproteína, $5,2 \mathrm{ng} / \mathrm{mL}$; con serologías para hepatitis $\mathrm{B}$ y hepatitis $\mathrm{C}$ negativas. $\mathrm{Al}$ examen físico se encontró una paciente con desnutrición, ascitis moderada y hepatomegalia. La tomografía abdominal contrastada demostró múltiples lesiones nodulares en ambos lóbulos hepáticos periféricos y con tendencia a confluir, sin hallazgos de cirrosis ni hipertensión portal, sin trombosis de las estructuras vasculares (figura 1). La sospecha clínica en una paciente de edad media sin cirrosis hepática fue descartar metástasis hepáticas, angiosarcoma y HEE. Se realizó biopsia hepática con estudios de anatomopatología e inmunohistoquímica, que fueron diagnósticos con HEE hepático (figura 2). La tomografía de tórax y la gammagrafía ósea sin enfermedad extrahepática. Por tratarse de un HEE hepático avanzado sin posibilidad de resección quirúrgica, se definió como opción de manejo el trasplante hepático, sin complicaciones en la cirugía y en el estudio del explante sin encontrarse compromiso vascular, ganglionar ni peritoneal. En el postrasplante presentó 2 episodios de rechazo celular agudo moderado tardío, tratados con bolos de esteroides. En la actualidad, 8 años después del trasplante, la paciente se encuentra en muy buen estado clínico, buena función del injerto hepático, sin recurrencia de la enfermedad de base y en tratamiento inmunosupresor con dosis bajas de ciclosporina y mofetil micofenolato.

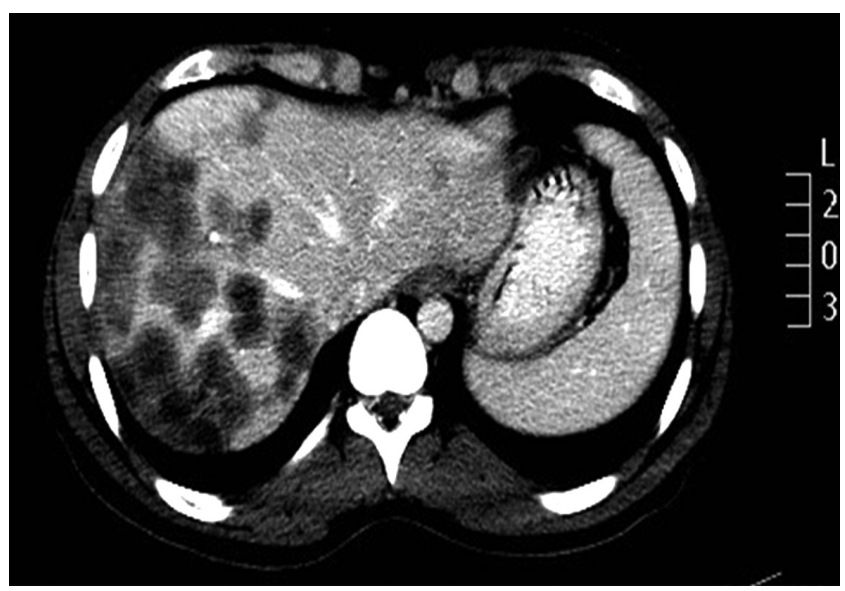

Figura 1. Múltiples nódulos hepáticos subcapsulares que tienden a la confluencia formando una masa hipodensa asociada con pérdida de volumen.

\section{Caso n. ${ }^{0} 2$}

Paciente de 21 años de edad, sexo masculino, sin antecedentes patológicos previos, quien consulta en el 2010 por sensación de masa y peso en el hipocondrio derecho, asociado con pérdida de peso. $\mathrm{Al}$ examen físico lo único relevante es el hallazgo de hepatomegalia. Las pruebas bioquímicas hepáticas reportan: AST, $46 \mathrm{U} / \mathrm{L}$; ALT, $36 \mathrm{U} / \mathrm{L}$; bilirrubina total, $0,8 \mathrm{mg} / \mathrm{dL}$; fosfatasa alcalina, $179 \mathrm{U} / \mathrm{L}$; gammaglutamil transpeptidasa, $111 \mathrm{U} / \mathrm{L}$; albúmina, $4,5 \mathrm{~g} / \mathrm{dL}$; alfa-fetoproteína, $2,43 \mathrm{ng} / \mathrm{mL}$; serologías para hepatitis B y hepatitis $C$ negativas. El ultrasonido abdominal reportó aumento del tamaño hepático, con presencia de lesiones nodulares en todo el parénquima. Se realizó tomografía 
abdominal contrastada y se encontraron lesiones bilaterales periféricas con patrón de realce en "diana" y retracción de la cápsula (figura 3). La principal posibilidad diagnóstica era metástasis hepáticas. Se llevó a biopsia hepática percutánea, en la que se encontró una histología compatible con HEE (figura 4), confirmado por inmunohistoquímica con marcadores CD31 y CD34 positivos (figura 5). Se llevó a cabo una búsqueda de enfermedad extrahepática con tomografía de tórax y gammagrafía ósea sin encontrar metástasis. Por tratarse de un tumor sin posibilidad de resección quirúrgica, se lleva el paciente a trasplante ortotópico de hígado, sin complicaciones en la cirugía ni el posoperatorio temprano. El estudio del explante (figura 6) confirmó el diagnóstico y descartó compromiso vascular, ganglionar y peritoneal. Un mes después del trasplante, el paciente presenta un episodio de rechazo celular agudo moderado, por lo que recibe reciclaje con metilprednisolona y ajuste de la inmunosupresión con buena respuesta clínica y bioquímica. Al momento de esta publicación, el paciente completa 4 años del trasplante con un injerto funcionante, sin recurrencia de la enfermedad y reingreso a la vida laboral.

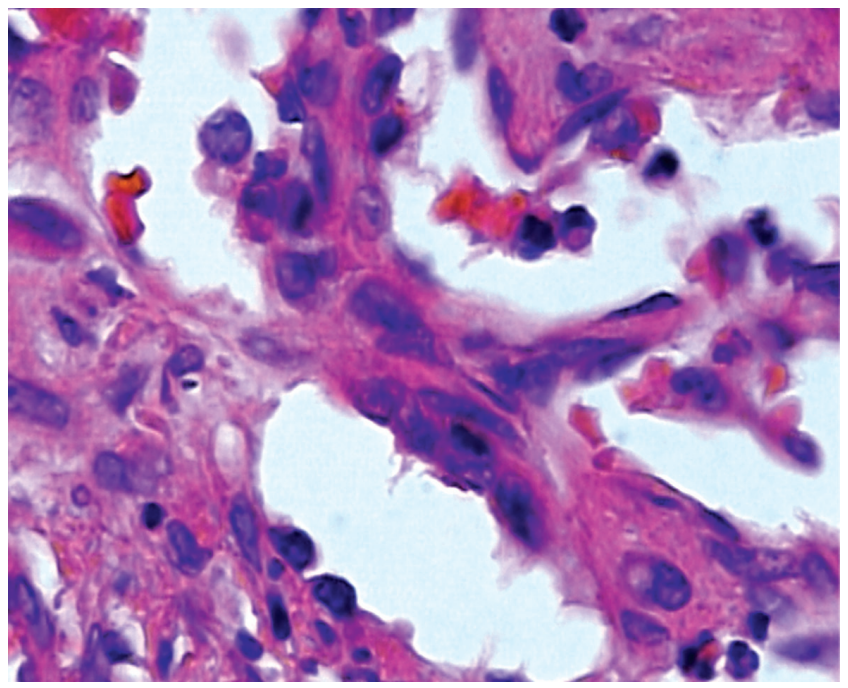

Figura 2. Coloración H\&E 400X. Espacios vasculares tapizados por células endoteliales prominentes, con núcleos hipercromáticos. En algunos espacios con grupos papilares de estas células hacia la luz.

\section{DISCUSIÓN}

El HEE hepático es un tumor de origen vascular con potencial comportamiento maligno. Es una enfermedad rara, con incidencia de solo 1 a 2 casos por cada millón de habitantes. Se presenta más frecuentemente en mujeres y el pico de edad es entre los 20 a 40 años $(1,2)$. Su etiología es desconocida, aunque se han relacionado con el consumo de anticonceptivos orales, la exposición al polivinil cloruro, la exposición al medio de contraste thorotrast y la infección crónica por hepa-

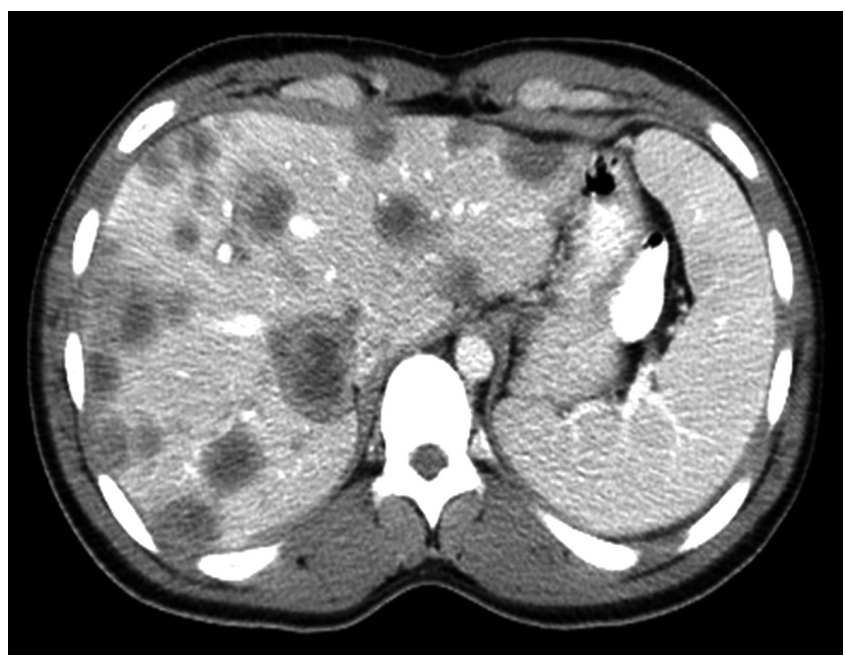

Figura 3. Lesiones nodulares con un patrón de realce en "diana”: parte central hipodensa que representa el estroma hialino y mixoide, anillo hiperrealzante interno por el incremento de la vasculatura y un halo externo avascular.

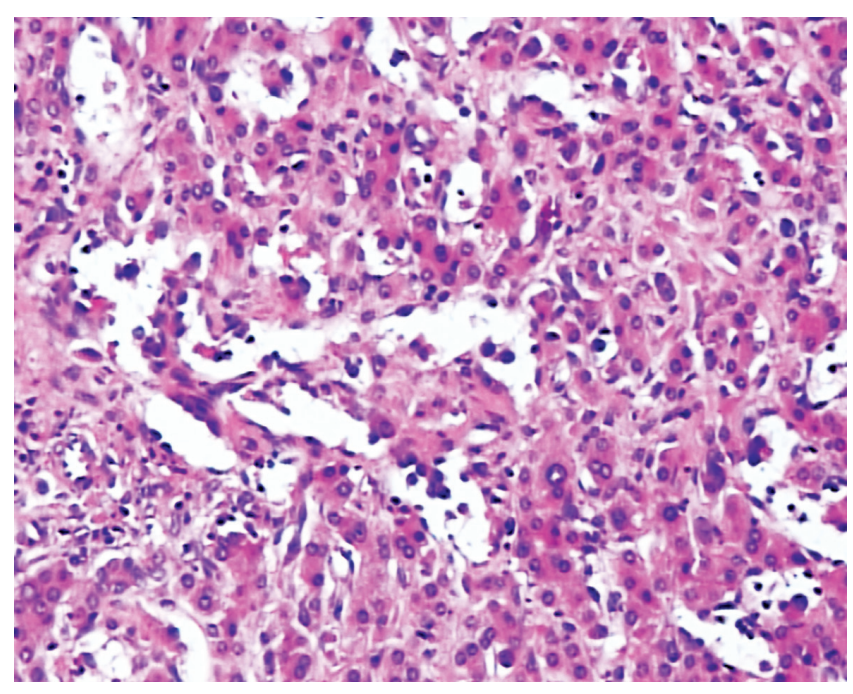

Figura 4. H\&E 200X. Parénquima hepático comprometido por la neoplasia. Los sinusoides son reemplazados por espacios vasculares soportados por tejido fibroconectivo. Las células neoplásicas revisten estos espacios.

titis B y C (3-5). Los síntomas más frecuentes son el dolor abdominal y la pérdida de peso, aunque en algunos casos los pacientes se encuentran asintomáticos o incluso pueden consultar con insuficiencia hepática aguda. El examen físico puede ser normal o mostrar ictericia, ascitis y hepatoesplenomegalia. En cuanto al diagnóstico, hay aumento de los niveles de fosfatasa alcalina y gamma-glutamil transferasa en el 50\% de los casos, los marcadores tumorales son negativos, incluida la alfa-fetoproteína, y el diagnóstico se sustenta en los estudios de radiología y patología. La tomografía axial 


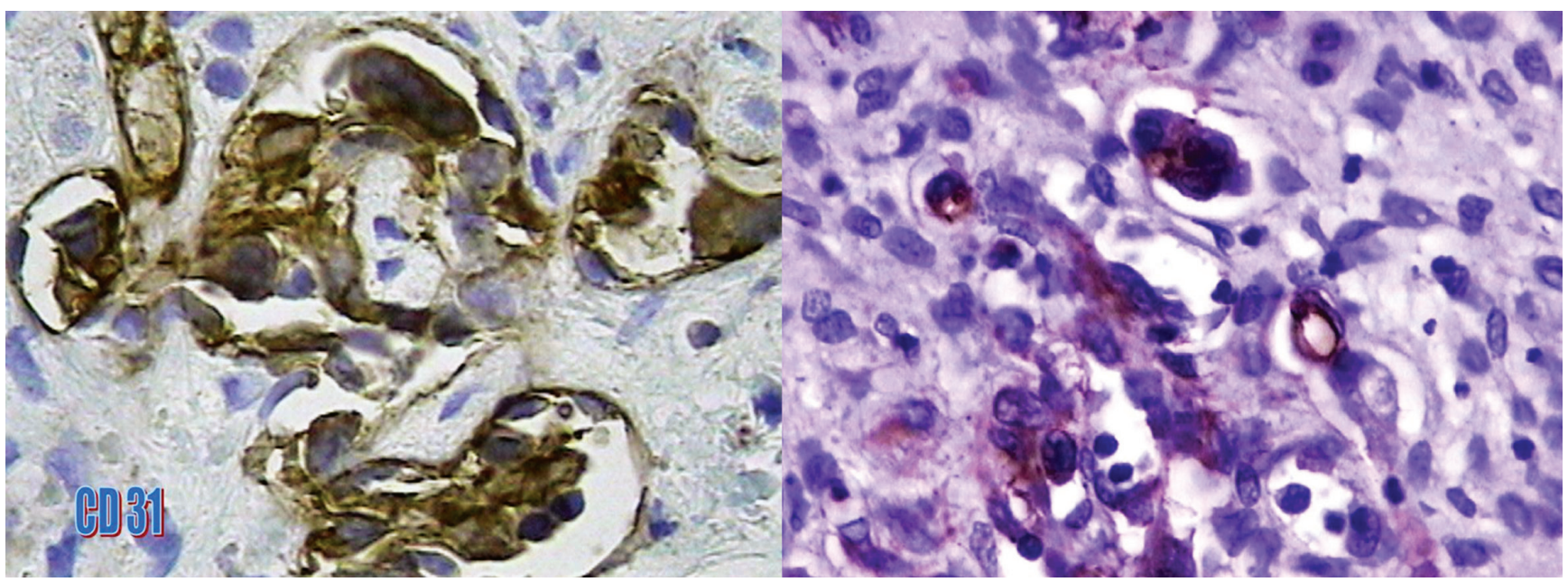

Figura 5. Inmunohistoquímica CD31/CD34 confirma el diagnóstico.
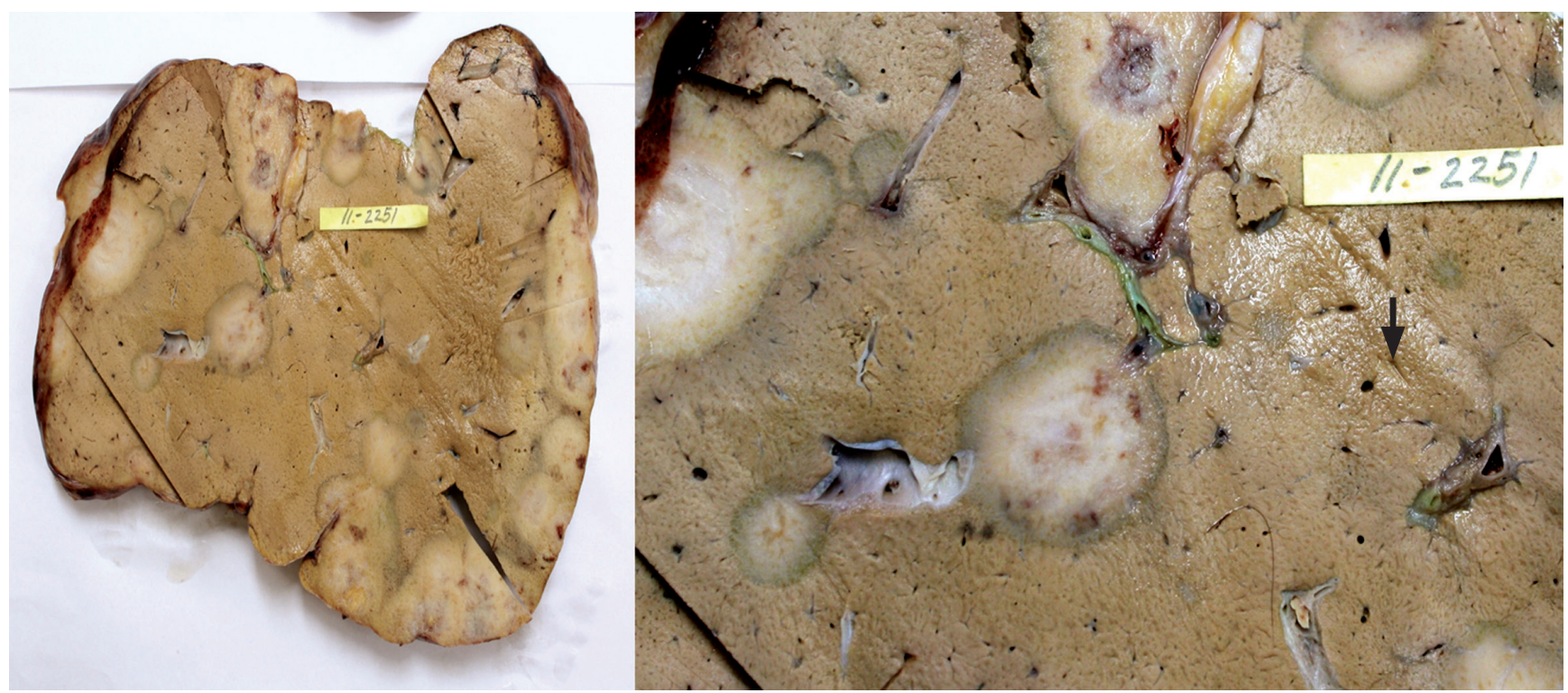

Figura 6. Foto macroscópica del hígado, con múltiples zonas de fibrosis, de color blanco que alternan con tejido hepático no cirrótico.

computarizada (TAC) y la resonancia nuclear magnética (RM) revelan lesiones nodulares, múltiples, bilaterales, periféricas, con retracción de la cápsula, con leve realce periférico del contraste. Se encuentran calcificaciones en el 20\% de las lesiones. Con el tiempo, las lesiones se unen formando una masa única que invade gran parte del hígado y habitualmente se asocia con trombosis portal tumoral. Son frecuentes las metástasis, encontrándose en el $40 \%$ de los pacientes al diagnóstico y los sitios más comunes son los pulmones, los ganglios linfáticos, el peritoneo, el hueso, el bazo y el diafragma (2). En todo paciente con sospecha de la entidad se debe realizar biopsia hepática, en la que se destacan como hallazgos principales un estroma fibrótico o mixomatoso y la presencia de células de características epiteliodes, con evidencia de diferenciación vascular por la presencia de eritrocitos en la luz. El diagnóstico no es fácil y debe diferenciarse del hemangioma esclerosante, el angiosarcoma, el colangiocarcinoma y el carcinoma metastásico, por lo cual estas biopsias deben ser evaluadas por patólogos expertos. Siempre debe confirmarse el origen endotelial con las tinciones de inmunohistoquímica CD31/CD34/FVIII-RAg $(2,7)$.

Con el diagnóstico ya establecido, el tratamiento de elección es la resección del tumor. El problema radica en que solo el $10 \%$ de los pacientes son susceptibles de resección quirúrgica en la evaluación inicial. La cirugía debe realizarse con fines oncológicos, ya que las resecciones parciales se asocian con crecimiento acelerado del tumor. Se han planteado otras estrategias de tratamiento como, por 
ejemplo, radioterapia, quimioterapia, terapia hormonal, embolización o quimioembolización, ablación percutánea y trasplante hepático, todos con diferentes tasas de éxito. En cuanto a la quimioterapia sistémica, en pacientes con enfermedad metastásica se ha usado agentes como la doxorrubicina, el 5-fluorouracilo y la vincristina, con sobrevida a 5 años menor del 20\%. La embolización o quimioembolización se usa como terapia puente para el trasplante hepático cuando los tiempos de espera son largos, sin que haya buen sustento científico para esta intervención. No existe ninguna utilidad de la radioterapia excepto con fines paliativos, por ejemplo, en metástasis pulmonares y óseas. El trasplante hepático surge entonces como la principal opción de tratamiento, teniendo en cuenta que casi el $90 \%$ de los pacientes se presentan con enfermedad de ambos lóbulos y, por tanto, sin posibilidad de resección. Cuando el trasplante hepático se ofrece a pacientes con tumores, la dificultad es el temor a la recurrencia. Sin embargo, como lo describieron Madariaga y colaboradores en una de las primeras series (1), la sobrevida de estos pacientes fue superior al $60 \%$ a 5 años, incluso en pacientes con compromiso vascular o ganglionar, siendo estos resultados mejores que los reportados por el mismo centro para carcinoma hepatocelular en dicha época. Los reportes más recientes confirman una sobrevida de los pacientes a 1, 5 y 10 años del $93 \%, 83 \%$ y $72 \%$, respectivamente, que es comparable con la sobrevida por etiologías no tumorales de pacientes sometidos a trasplante hepático (9). Estos resultados han cambiado la percepción mundial con respecto al trasplante de hígado para los pacientes con HEE (2).

En los estudios de los pacientes trasplantados, la edad promedio es 30 años, con predominio del sexo femenino, la mayoría sin comorbilidades $(9,10)$. Estas características son factores que favorecen los buenos resultados postrasplante hepático. El 19\% de los pacientes son asintomáticos al momento del trasplante. Lo anterior resalta que independientemente de la presentación clínica, el compromiso tumoral hepático es el que define la indicación del trasplante (9). A pesar de que el 50\% de los casos se presentaba con enfermedad avanzada e invasión vascular, y que este hallazgo se comportó como un factor adverso para la recurrencia, no está contraindicado el trasplante hepático y debe analizarse cada caso en particular. En el 17\% de los pacientes se halló enfermedad extrahepática, que incluía compromiso ganglionar, aunque sin afectar la sobrevida a largo plazo.

En cuanto a la cirugía, hay que resaltar que en la mayoría de los casos se realizó el procedimiento estándar, reportándose menor dificultad técnica por la ausencia de hipertensión portal y menor número de transfusiones sanguíneas. Las intervenciones quirúrgicas previas no impactaron negativamente los resultados del trasplante. La recurrencia de la enfermedad tumoral se presenta del $20 \%$ al $38 \%$ de los pacientes y esta es la principal causa de muerte en estos individuos. Los pacientes con recurrencia deben tratarse en forma agresiva con quimioterapia sistémica, medicamentos antiangiogénicos y el uso de inmunosupresores tipo mTor, aunque debe aclararse que los anteriores tratamientos todavía no tienen soporte en ensayos clínicos.

\section{CONCLUSIÓN}

En conclusión, el hemangioendotelioma epiteliode hepático debe sospecharse en todo paciente joven no cirrótico que consulte con nódulos hepáticos múltiples, bilaterales, periféricos y con patrón de realce en "diana". A pesar de que el tratamiento de elección es la resección hepática, esta no puede realizarse sino en un pequeño grupo de pacientes y, por tanto, el trasplante hepático aparece como la principal opción de tratamiento, incluso en pacientes con enfermedad avanzada.

\section{REFERENCIAS}

1. Madariaga J, Marino IR, Starzl TE, et al. Long-term results after liver transplantation for primary hepatic epithelioid hemangioendothelioma. Ann Surg Oncol. 1995;2(6):483-7.

2. Primary malignant hepatic epithelioid hemangioendothelioma, comprehensive review of the literature with emphasis on the surgical therapy. Cancer. 2006; 107:2108-21.

3. Dean PJ, Haggitt RC, O’Hara CJ. Malignant epithelioid hemangioendothelioma of the liver in young women. Relationship to oral contraceptive use. Am J Surg Pathol. 1985;9:695-704.

4. Gelin M, Van de Stadt J, Rickaert F, De Prez C, Levarlet M, Adler M, et al. Epithelioid hemangioendothelioma of the liver following contact with vinyl chloride. Recurrence after orthotopic liver transplantation. J Hepatol. 1989;8:99-106.

5. Lauffer JM, Zimmermann A, Krahenbuhl L, Triller J, Baer HU. Epithelioid hemangioendothelioma of the liver. A rare hepatic tumor. Cancer. 1996;78:2318-27.

6. Weiss SW, Enzinger FM. Epithelioid hemangioendothelioma: a vascular tumor often mistaken for a carcinoma. Cancer. 1982;50:970-81.

7. Fujii T, Zen Y, Sato Y, Sasaki M, Enomae M, Minato H, et al. Podoplanin is a useful diagnostic marker for epithelioid hemangioendothelioma of the liver. Mod Pathol. 2008;21:125-30.

8. Makhlouf HR, Ishak KG, Goodman ZD. Epithelioid hemangioendothelioma of the liver: a clinicopathologic study of 137 cases. Cancer. 1999;85:562-82.

9. Lerut JP, Orlando G, Adam R, Schiavo M, Klepnauer J, Mirza D, et al. The place of liver transplantation in the treatment of hepatic epitheloid hemangioendothelioma. Report of the European Liver Transplant Registry. Ann Surg. 2007;246:949-57.

10. Rodriguez JA, Becker NS, O’Mahony C, et al. Long-term outcomes following liver transplantation for hepatic hemangioendothelioma: The UNOS Experience from 1987 to 2005. J Gastrointest Surg. 2008;12:110-6. 